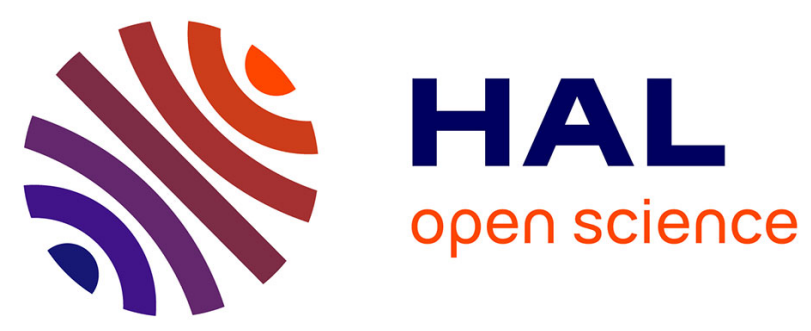

\title{
Optimal Dynamic Line Rating Forecasts Selection Based on Ampacity Probabilistic Forecasting and Network Operators' Risk Aversion
}

\author{
Romain Dupin, Andrea Michiorri, Georges Kariniotakis
}

\section{To cite this version:}

Romain Dupin, Andrea Michiorri, Georges Kariniotakis. Optimal Dynamic Line Rating Forecasts Selection Based on Ampacity Probabilistic Forecasting and Network Operators' Risk Aversion. IEEE Transactions on Power Systems, In press, pp.1-1. 10.1109/TPWRS.2018.2889973 . hal-01998856

\section{HAL Id: hal-01998856 https://hal.science/hal-01998856}

Submitted on 12 Feb 2019

HAL is a multi-disciplinary open access archive for the deposit and dissemination of scientific research documents, whether they are published or not. The documents may come from teaching and research institutions in France or abroad, or from public or private research centers.
L'archive ouverte pluridisciplinaire HAL, est destinée au dépôt et à la diffusion de documents scientifiques de niveau recherche, publiés ou non, émanant des établissements d'enseignement et de recherche français ou étrangers, des laboratoires publics ou privés. 


\title{
Optimal Dynamic Line Rating Forecasts Selection Based on Ampacity Probabilistic Forecasting and Network Operators' Risk Aversion
}

\author{
Romain Dupin, Andrea Michiorri, Member, IEEE, and George Kariniotakis, Senior Member, IEEE
}

\begin{abstract}
Real-time current-carrying capacity of overhead conductors is extremely variable due to its dependence on weather conditions, resulting in the use of traditionally conservative static ratings. This paper proposes a methodology for exploiting the latent current-carrying capacity of overhead transmission lines taking into account line ampacity forecasts, power flow simulations and the network operator's risk aversion.

The procedure can be described as follows: Firstly, probabilistic forecasts for the current rating of transmission lines are generated, paying particular attention to the reliability of the lower part of the distribution. Secondly, a cost benefit analysis is carried out by solving a bilevel stochastic problem that takes into account the reduction in generation costs resulting from a higher power transfer capacity and the increased use of reserves caused by forecast errors. The risk appetite of the network operator is considered in order to accept or penalize high-risk situations, depending on whether the network operator can be described as risk neutral or risk averse.
\end{abstract}

Index Terms-- Dynamic Line Rating, Numerical Weather Predictions, Probabilistic Forecasts, Risk Management

\section{INTRODUCTION}

Ous UURENTLY, power system operators face several challenges, due to increased peak demand, electricity market deregulation and increasing penetration of renewable energy sources. Due to these latter factors, grid sections are often operated closer to their voltage or current limits. From a network operator's point of view, network reinforcement is often the default option for alleviating these problems. However, this is a capital-intensive option and, in this context, the alternative solution of the Dynamic Line Rating (DLR) is gaining popularity.

DLR technology centres on the fact that the current-carrying capacity of overhead conductors is limited by thermal constraints, in addition to voltage drops and stability limits. When the operating temperature rises, the conductors elongate, approaching the ground or other objects. When the clearance distance between the line and the objects below is too short this can lead to potentially dangerous situations. In order to limit disastrous consequences, a minimum clearance is determined

Part of this work was carried out thanks to an exchange mobility grant awarded to the first author from the project ELECTRA IRP (Grant No 609687), funded in part by the European Commission under the 7th Framework Programme.

R. Dupin, A. Michiorri and G. Kariniotakis are with MINES ParisTech, PSL Research University, PERSEE - Centre for processes, renewable energies and by national or grid regulations, including the definition of a maximum temperature for the core of the line. Conductor temperature is in turn dependent on the heat balance established between the heat dissipated by the Joule effect within the conductor, and the heat exchange with the environment on its external surface. This heat exchange is strongly influenced by wind speed, air temperature and solar radiation, parameters that can vary considerably in the space of one day or even one hour. The link between the line temperature and weather parameters, which is described in detail in [1], [2], is used to set a current rating in line with regulations .

Traditionally, the parameters used to define the line rating are set as static. The current rating obtained is called Static Line Rating (SLR), which is usually defined so that it remains below the real-line rating up to $99 \%$ of the time [3] for the duration of a season. Based on this definition, a significant margin of improvement exists between SLR and the real value of the line rating, depending on weather characteristics. In this context, it was initially proposed in [4] to set the line rating according to dynamic measures of weather parameters, the new rating being qualified as the DLR.

The use of DLR instead of SLR for network operations presents multiple benefits and has already been studied in the literature. For example, [5] and [6] show the potential impact of DLR on the economic dispatch, [7] shows how DLR can contribute to increasing renewable energy penetration without grid reinforcement, and [8] demonstrates how grid reliability can be improved with DLR. These aspects could be taken into account during planning operations, in which case DLR forecasts would be required.

The majority of applications proposed in the literature feature the generation of probabilistic DLR forecasts. Most of the time, based on these forecasts, a conservative choice of an ampacity value is made for use in planning operations, and quantile forecasts with a fixed nominal proportion $\tau$ are regularly employed [9]. Forecasts $\widehat{D L R}_{t+h \mid t}^{\tau}$ made at an instant $t$ for an instant $\mathrm{t}+\mathrm{h}$ are then set to ensure the probability of being higher than the future observation $D_{L R}+h$ equal to $\tau$ :

$$
\mathrm{P}\left(\widehat{\operatorname{DLR}}_{\mathrm{t}+\mathrm{h} \mid \mathrm{t}}^{\tau}>\mathrm{DLR}_{\mathrm{t}+\mathrm{h}}\right)=\tau
$$

energy systems, CS 10207, 1 rue Claude Daunesse 06904 SophiaAntipolis Cedex, France.

(e-mails: romain.dupin, andrea.michiorri, georges.kariniotakis each with @ mines-paristech.fr) 
A state of the art of real applications of this kind in Europe is described in [10]. In [11] and [12], where the experiences of the NETFLEX project are described, probability $\tau$ is set as equal to $2 \%$. In [13], probability $\tau$ fixed levels of $1 \%, 5 \%, 10 \%$ and $20 \%$ are tested. In [14], a probability $\tau$ of $1 \%$ is considered. Other studies could also be quoted, such as [15] and [16], where $\tau$ probabilities of $10 \%$ and $2.5 \%$ are respectively considered.

Recently, it has been proposed in the literature to no longer set the value of DLR forecasts with a fixed level of frequency, but to use stochastic optimization instead. In [17] and [18], this is done with two-stage stochastic optimization, which aims at minimizing the sum of the generation costs, reserve allocation and potential reserve activation due to DLR forecast errors. A similar approach is also proposed in [19], where instead of reserve activation, it is considered that the retroactions are achieved through voluntary and involuntary load reduction. References [20] and [21] both propose considering that the constraint associated with the line ampacity could be replaced with a cost in the objective function in case of non-respect of the constraint.

The above two approaches are radically different. The one with the fixed quantiles favours a conservative approach independent from opportunities to improve social welfare, while ensuring a low risk level. On the contrary, with the second approach based on stochastic optimization, the authors consider a risk-neutral approach and aim at maximizing the social welfare. In this context, the term "social welfare" refers to the sum of the consumer and producer surpluses. If the load is considered as inelastic towards prices, maximizing the social welfare is equivalent to minimizing the operation costs.

In this paper, we propose to evaluate the benefits of DLR based on an intermediate strategy, which aims at improving social welfare while ensuring low levels of risk, required for application on the field. The key contributions can be summarized as follows:

1) Several alternative strategies for integrating DLR into planning operations are proposed. They aim at improving the economic benefits, (referred to here also as the "social welfare") for a case where electricity consumption is inelastic towards price, while maintaining low values for several parameters to which the Transmission System Operator (TSO) is averse. The evaluation of these strategies allows us to understand the significant benefits of using risk-averse strategies for DLR probabilistic forecasts, compared to traditional approaches that use fixed quantiles or risk-neutral strategies.

2) We propose a test case and a methodology to evaluate the economic value of both DLR forecast models and proposed strategies for using DLR forecasts. This is based on the use of lookup table and allow considering grid characteristics.

3) The proposed study takes into account a high number of realistic DLR probabilistic forecasts, generated with weather station observations and machine learning methods. This is a more complete approach than similar ones proposed in the literature, which generally consider only a single virtual DLR probabilistic forecast.

The paper is structured as follows: first, in section II, the proposed methodology is presented. Section III presents the case study used for the model evaluation. In section IV the main results are presented, and finally section $\mathrm{V}$ presents the conclusions and perspective of this work.

\section{MODELLING APPROACH}

The problem to be solved is the optimal operation of a grid on which some lines are equipped with DLR. It is similar to that formulated in [18] and [22], which assess the DLR value in grid management. Since several uncertainties are involved, a stochastic optimisation approach is applied. Below, several problem formulations are proposed for setting the DLR forecasts, according to different objectives set by the TSO.

\section{A. Risk Neutral Strategy - Vertically Integrated Monopoly}

We first consider that the electricity producers and electric system operators are the same actor. The problem addressed here is described in [18] and can be modelled as a two-stage stochastic optimization problem.

For a stochastic optimization problem taking into account DLR forecasts, different terms could be considered in the objective function. One important question concerns the introduction and use of penalties regarding situations in which the current is slightly higher than the thermal limits of the lines, as in [20], [21], [23]. Such cost definitions require knowledge of potential incidents on the grid, their associated probability, and recourse actions when these costs are too high. In this paper, we consider a preventive approach regarding N-1 incidents, and penalties requiring a corrective approach are not introduced. It should be stressed that the simplifications allowed with this preventive approach permits the use of lookup tables which are necessary to provide computations for a high number of observations in a reasonable time.

The objective function is risk neutral, the objective being to minimize production costs of generators in the system plus the costs of reserve allocation and potential activation (2):

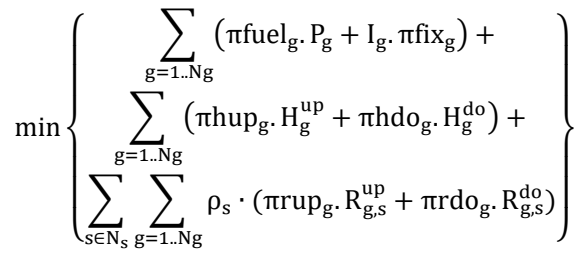

where $\mathrm{Ng}$ is a set of conventional generators; $I_{g}$ is a binary variable with value 1 describing a committed generator, and 0 if not; $\pi f_{u e l} g_{g}$ is the fuel cost for generator $g(€ / M W h) ; \pi f i x_{g}$ is the commitment price for a conventional generator $\mathrm{g}(€ / \mathrm{h}) ; \mathrm{P}_{\mathrm{g}}$ is the scheduled output of generator $\mathrm{g}(\mathrm{MW})$; $^{\mathrm{m} u \mathrm{p}_{\mathrm{g}}}$ and $\pi \mathrm{hdo} \mathrm{g}$ are the costs for maintaining up and down reserve for a generator $\mathrm{g}(€ / \mathrm{MWh}) ; \mathrm{H}_{\mathrm{g}}^{\mathrm{up}}$ and $\mathrm{H}_{\mathrm{g}}^{\mathrm{do}}$ are the up and down reserve service holding amounts for generator $\mathrm{g}(\mathrm{MW}) ; \mathrm{N}_{\mathrm{s}}$ is the set of potential future realizations of DLR and other stochastic variable observations, with each scenario having a probability $\rho_{s}$ of occurrence set with probabilistic forecasts; $\pi_{r u p}$ and $\pi r d o_{g}$ are the reserve activation costs ( $\left.€ / M W h\right)$; and $R_{\mathrm{g}, \mathrm{s}}^{\mathrm{up}}$ and $\mathrm{R}_{\mathrm{g}, \mathrm{s}}^{\mathrm{do}}$ are the activated reserves from a generator $\mathrm{g}$ at scenario $\mathrm{s}$ (MW).

The constraints are those of a DC power flow, and are described in [18] and [22]. Here, N-1 constraints are added to 
account for the risks of line failure, as proposed in [24], which investigates DLR use for transfer capacity setting.

\section{B. Risk-Neutral Strategy - Separate Grid Operators and Electricity Producers}

In the previous strategy, the generation planning is affected by the potential recourse actions set by the TSO. It is however unlikely that electricity producers would modify their dayahead decisions according to the risks and potential costs of DLR forecast errors, which are assumed by the TSO.

In order to decouple the decision of the generator-planned production levels from the decision of the DLR forecast value, a bilevel stochastic optimization problem is used here, with a leader problem (3)-(4) and a follower problem (5):

$$
\min _{x \in X, y \in Y} F(x, y)
$$

s.t.

$$
\begin{aligned}
& G_{i}(x, y) \leq 0 \text { for } i \in\{1,2, \ldots, I\} \\
& y \in \arg \min _{z \in Y}\left\{f(x, z): g_{j}(x, z) \leq 0, j \in\{1,2, \ldots, I\}\right\}
\end{aligned}
$$

For a risk-neutral approach, the upper-level objective function $\mathrm{F}$ is the same as that described in (2), and the constraints $\mathrm{G}_{\mathrm{i}}$ are those described in the previous section.

The lower-level objective function $\mathrm{f}$ is the objective function defined according to the goals of the electricity producers. Considering no uncertainties except those for which the TSO is responsible and which in this case are only the DLR forecast errors, this can be written as (6):

$$
f(x, z)=\left\{\sum_{g=1 . . N g}\left(\pi f^{\prime} \mathrm{fl}_{\mathrm{g}} \cdot \mathrm{P}_{\mathrm{g}}+\mathrm{I}_{\mathrm{g}} \cdot \pi \mathrm{fix} \mathrm{g}\right)\right\}
$$

In a future work, reserve activation and allocation terms could be added in (6), provided that other uncertain variables such as renewable energy levels of production are added, for which the producers assume the forecast error risks. The constraints $g_{j}$ are the same as those of the leader problem, minus the constraints associated with the values of reserve allocations and activations. The upper-level decision vector includes the values of allocated reserves, activated reserves and DLR forecasts. The lower-level decision vector includes the planned production levels and the list of activated generators. Only the DLR forecast value, set with the leader problem, has an impact on the constraints of the follower problem.

\section{Risk Averse Strategy}

In the previous section, the objective function (2) was defined such that the TSO was considered risk neutral in terms of reserve activation risks. Here, we propose to modify this problem by introducing a risk appetite function $\mathrm{v}_{\beta}(\mathrm{x})$, to take into account TSOs' risk aversion:

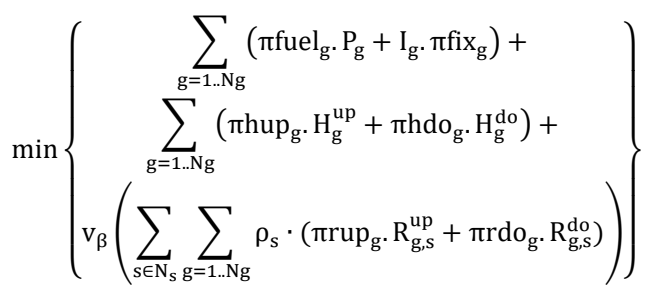

It should be stressed that the risk aversion towards risk activation costs defined here is a trade-off solution: risk aversion towards other parameters, such as load shedding, would be more representative of TSOs' behaviour. However, it would make the problem more complex, and impossible to compute for a very high number of scenarios and forecasts, as it is the case here. By considering these reserve costs as similar to risk indices, it is thus possible to evaluate first different risk aversion functions, and also determine the economic value of DLR forecast models in a latter approach.

The shape of the function $\mathrm{v}_{\beta}$ is arbitrarily set by the TSO, depending on its objectives. Here, three functions are proposed inspired from [20], [21], where virtual costs associated with line ampacity violations by currents are defined:

- A linear function:

$$
\mathrm{v}_{\beta}(\mathrm{x})=(1+\beta) \cdot \mathrm{x} \quad \beta \geq 0
$$

- A quadratic function:

$$
v_{\beta}(x)=x+\beta \cdot x^{2} \quad \beta \geq 0
$$

- An exponential function:

$$
v_{\beta}(x)= \begin{cases}x & \text { if } \beta=0 \\ \frac{e^{\beta \cdot x}-1}{e^{\beta}-1} & \text { if } \beta>0\end{cases}
$$

The parameter $\beta$ aims at describing the TSO's aversion to the risk of observing a DLR forecast error with a high cost. When $\beta$ is set equal to 0 , the TSO exhibits risk-neutral behaviour.

\section{Simulation Approach}

The solution of the problem described above represents a significant computational challenge due to the high number of constraints generated by the stochastic nature of the problem, the respect of the N-1 criterion, and the presence of discrete values related to the activation of generators. This paper aims at carrying out a prospective analysis of the benefits of different DLR forecast strategies over a long period of time and considering a high number of different parameters, here the values of $\beta$ and the nature of the penalty functions.

This requires solving a high number of stochastic problems. As an example, considering 100 values of $\beta, 45000$ forecasts and three different penalty functions, 13.5 million simulations would be required. It is therefore necessary to reduce the computational time taken to resolve the optimisation problems.

To do so, the problem is simplified considering the following hypotheses and properties:

- Since the DLR forecast may remain relatively close to the SLR, and that up and down reserves must be allocated in case of any forecast error scenarios, it is unlikely that DLR would have a significant impact on the list of activated generators. The list of activated generators $I_{g}$ is thus defined considering that all DLR values are equal to the SLR.

- For the case presented in this paper, only line failures and DLR forecast uncertainties are considered. Uncertainties related to renewable energy sources and electric demand are not considered. Recourse actions involving activating reserves can only be carried out for inaccurate DLR forecasts, while other potential incidents (which may face a similar preventive approach) are outside the scope of this work. 
For a test case such as the one described in [18], the property of reversibility could be considered regarding the states of generator production towards DLR values, where all conventional generators participate in recourse actions, and the reserve activation costs are proportional to the day-ahead prices. With the properties described above, the problem can be resolved by solving different Security Constrained Optimal Power Flows (SCOPF) according to the following steps:

1) A first SCOPF is resolved to initialize the problem, where the line ratings are set at the value that corresponds to the SLR, to define the list of the activated generators $I_{g}$.

2) After setting $I_{g}$, a SCOPF is computed for each possible value of line rating $\Delta_{\mathrm{l}}$.

3) For each forecast $\Delta_{\mathrm{l}}$, the values of allocated reserves $\mathrm{H}_{\mathrm{g}}^{\mathrm{up}} / \mathrm{H}_{\mathrm{g}}^{\mathrm{do}}$ are set by: a) running a SCOPF with a line rating set as $\Delta_{\mathrm{l}}$, b) calculating the difference between generator outputs in this situation and generator outputs after the SCOPF in step 1. This method implies that the problem associated with the SCOPF is strictly convex, which may not be the case with a linear approach. In the study proposed here, a negligible quadratic component is added to the generator cost function, in order to ensure strict convexity.
4) For a scenario $s$ associated with a DLR realization $\Delta_{\mathrm{l}, \mathrm{s}}$, if $\Delta_{\mathrm{l}, \mathrm{S}}$ is lower than inferior to the DLR forecasted value $\Delta_{\mathrm{l}}$ selected by the TSO, the activated reserves are found by computing the differences in the outputs of the different generators associated with the solutions of the SCOPFs made with the line ratings equal to $\Delta_{\mathrm{l}, \mathrm{s}}$ and $\Delta_{\mathrm{l}}$.

5) Then, considering the levels of probability $\rho_{s}$ of a DLR observation $\Delta_{\mathrm{l}, \mathrm{s}}$, it is possible to select an optimal value for the DLR forecast $\Delta_{\mathrm{l}}$.

By considering a limited set of possible states of load L1 and L2 (in this work, 20 for each load area, going from $0 \%$ of the nominal load to $100 \%$ ), DLR planned forecasts (100, going from $100 \%$ of the SLR to $200 \%$ ) and DLR potential observations (100, going from $100 \%$ of the SLR to $200 \%$ ), it is possible to compute the different SCOPFs for each state in which DLR improvements bring benefits. Thus, lookup tables can be built (Fig. 1), each value of the table being associated to a tuple of scenario, and the use of this tool allows reducing considerably the computation time for a period associated to a high number of observations (Fig. 2).

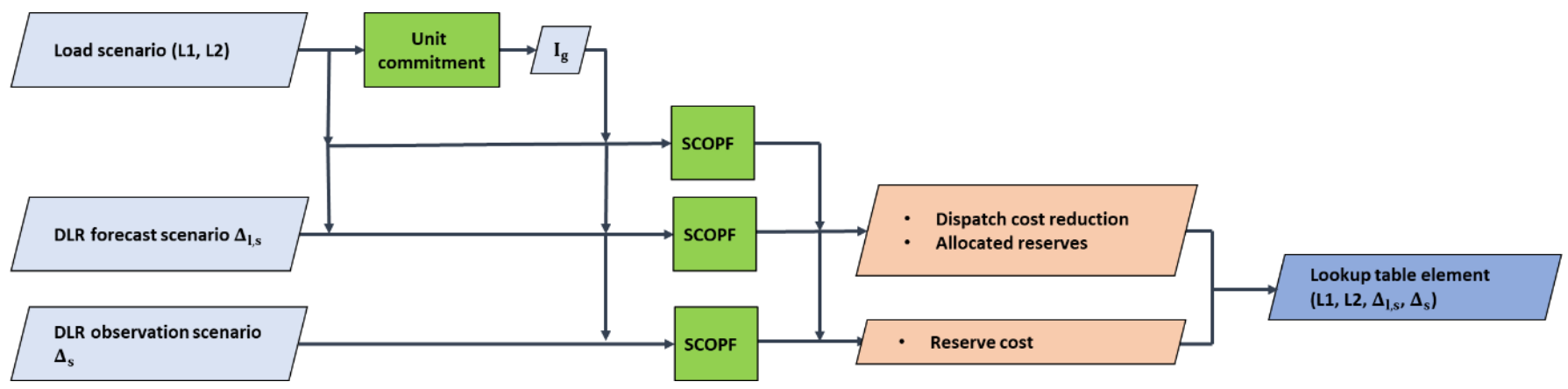

Fig. 1. For a load scenario (L1, L2), a DLR forecast $\Delta_{\mathrm{l}}$ and a DLR observation $\Delta_{\mathrm{l}, \mathrm{s}}$, computation process for setting the associated value in the lookup table.

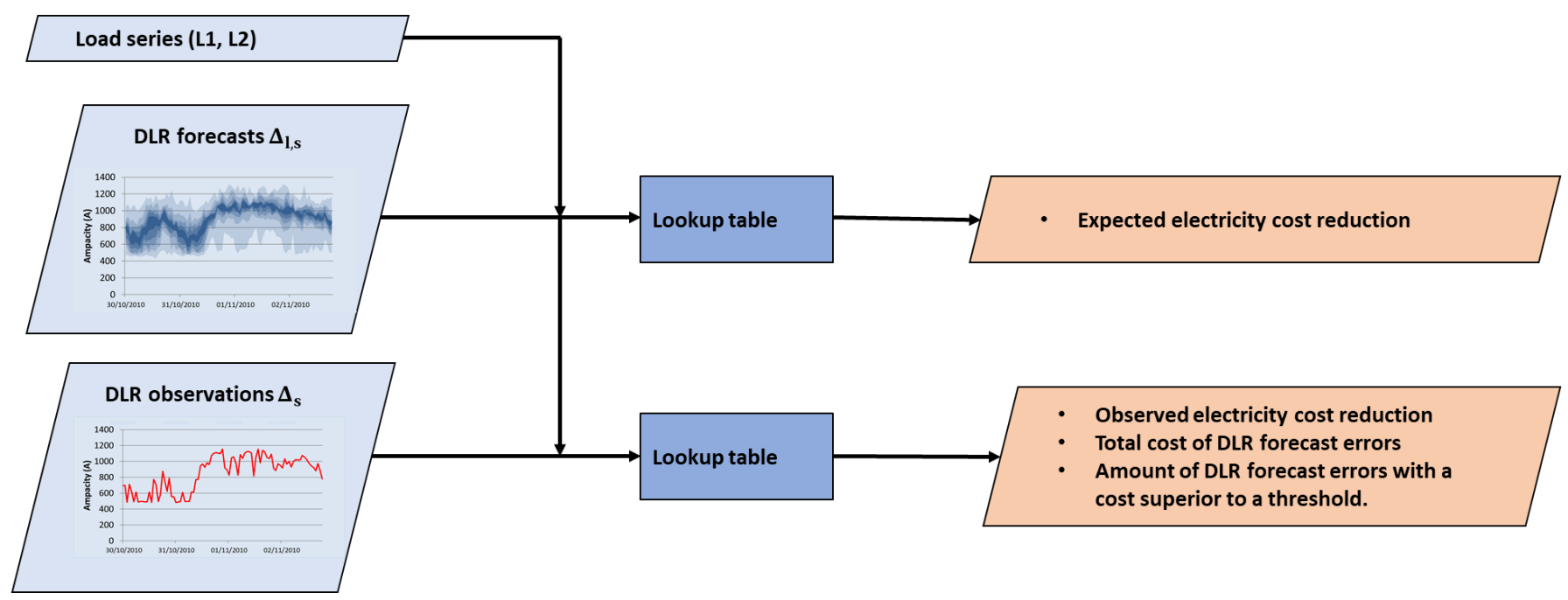

Fig. 2. Flowchart associated to the computations made with the lookup tables. 


\section{E. DLR Forecast Generation}

Here we use the same weather station data as in [15]. For a line located in the UK, several weather stations have been installed all along the line for DLR use. In this study we consider 6 of them. For each station, weather characteristics were measured every 5 minutes for the period 2009-2010. Using Numerical Weather Predictions (NWP) provided by the European Centre for Medium-Range Weather Forecasts (ECMWF), day-ahead probabilistic forecasts are generated using a machine learning method.

The available data set is split into training and testing sets. For this study, these sets are generated with sliding windows, the models are updated every month, and the training sets used correspond to observations associated with the whole year preceding the moment of the model update.

The models are trained to provide forecasts every day at 12:00 for horizons going from 24 hours to 47 hours. The following inputs are used to generate the forecasts:

- The DLR observation at the instant $t$ the forecast is made.

- The mean value of the DLR observations made at the instants $\mathrm{t}+\mathrm{h}-48$ hours, $\mathrm{t}+\mathrm{h}-72$ hours and $\mathrm{t}+\mathrm{h}-96$ hours.

- NWPs for the zonal and meridional wind speeds at $10 \mathrm{~m}(\mathrm{U}$ and V), the surface solar radiation downwards (S) and the ambient temperature at $2 \mathrm{~m}(\mathrm{~T})$ for the four positions that frame the location of the weather station. The spatial resolution of the considered NWPs is $0.125^{\circ}$. Since the ECMWF forecasts are only made for 3-hour time steps and DLR forecasts are requested with hourly resolution, linear interpolation is applied to obtain hourly values.

- Four DLR forecasts associated with the ECMWF forecasts are provided at the instant where the forecasts are made for the considered horizon. For a given position, the DLR forecast is provided with the CIGRE physical model [1] using the forecast values of $\mathrm{U}, \mathrm{V}, \mathrm{S}$ and $\mathrm{T}$.

No selection of the transmission lines' critical span is made here. Thus, the data from each weather station are studied in the same way as if each station were set at the position of the critical span as proposed in [15] in order to increase the amount of observations used.

Regarding the used machine learning method, a Quantile Regression Forest model (QRF) is selected [25]. This model is the same as the one used in [15] for DLR forecasting. This machine learning method is an ensemble method, based on the generation of $\mathrm{k}$ decisional trees, each one trained with a randomly selected subset of features and of data. For the forecast generation, the outputs of all the trees are concatenated and sorted, and the quantile forecasts are drawn from the sorted list (Fig. 3).

The choice of such a machine learning method could be discussed. Regarding the latest competition associated to the use of machine learning in energy fields, the Global Energy Forecasting Competition [26], the methods using bootstrap aggregating processes (bagging) as the QRF appear as being less effective than the ensemble methods associated to boosting, as the XGBoost model [27]. Compared to such methods, the
QRF tends to generate bias errors, the model being less effective at explaining complex relationships, and even if such methods have not been used yet to provide DLR forecasts, a XGBoost model or a Light Gradient Boosting Machine model [28] could be preferred. However, for this case study, the differences between the two models would be little, due to the little amount of features being used. Moreover, a QRF is easy to configure, the model outputs converging when the number of decisional trees becomes high, and a single QRF directly provides all the quantile forecasts. On the contrary, with gradient boosting methods, a different model should be trained for each quantile, and methods as Bayesian optimization [29] and cross validation should be used for each model in order to avoid errors due to a non-optimal configuration, thus making the training of the model much longer. To give an order of magnitude, instead of a single QRF with the bagging method, 999 models would be required considering boosting, in addition to a high number of models being trained for each one to provide optimal parameters. Due to these reasons, the QRF is here preferred to gradient boosting methods.

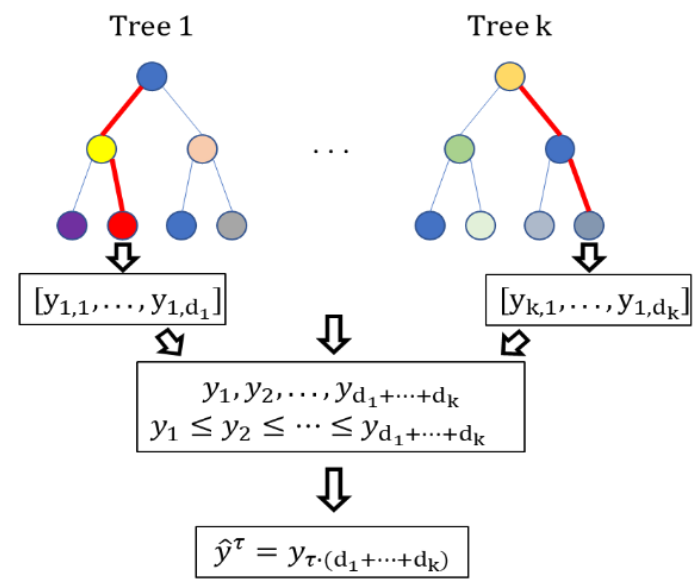

Fig. 3. Illustration of the process of a quantile forecast generation with a QRF method.

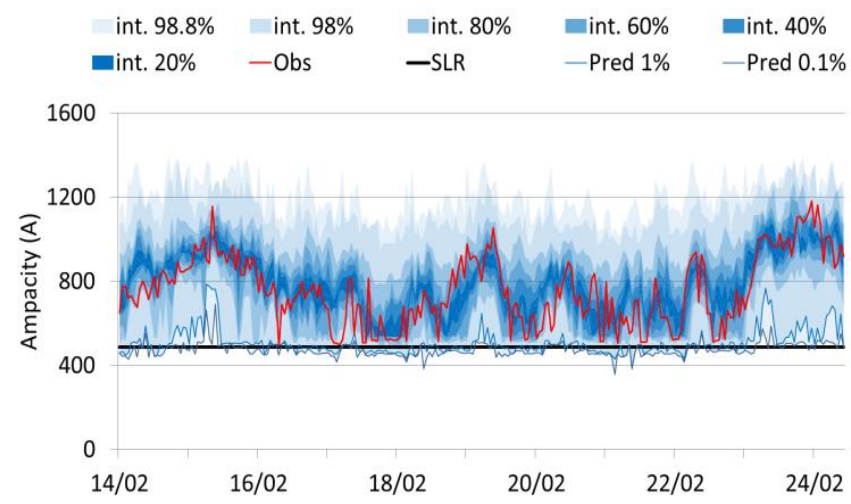

Fig. 4. Example of day-ahead DLR probabilistic forecasts for station 1, the different probability intervals (int.) being shown. The $0.1 \%$ and $1 \%$ quantile forecasts (Pred.) are the lower limits of the $99.8 \%$ and $98 \%$ probability intervals.

This model is set up with 2000 trees, each of which is trained with 5 randomly selected features and with a maximal leaf size 
set as equal to 5 . In most of the studies associated with DLR forecasts, the forecasts are used with fixed quantiles varying from $1 \%$ to $10 \%$, which thus requires using the forecast model to provide percentile forecasts. However, here, these forecasts are used as inputs of risk-averse strategies, which aim at providing results that are similar to $1 \%$-quantile DLR forecasts in terms of risks. For this reason, the model provides a set of 999 quantile forecasts, for quantiles ranging from $0.1 \%$ to $99.9 \%$ in $0.1 \%$ steps. An example of such forecasts is shown in Fig. 4.

The Mean Absolute Percentage Error (MAPE) and reliability criteria are considered here to evaluate the performance of the forecast models with regard to state-of-the-art models. First, regarding the MAPE, a comparison can be made with the persistence model, i.e. a model where a forecast is set as equal to the observations made at the moment of the forecast.

For this case, the MAPE for the QRF and the persistence model are equal to $11.1 \%$ and $16.1 \%$ respectively. The relative difference of $31 \%$ is in line with the results found in [15].

In terms of reliability, the frequency of overestimation for quantiles $0.1 \%, 1 \%$ and $2 \%$ is equal to $0.8 \%, 1.8 \%$ and $2.7 \%$ respectively. With binomial laws, considering perfectly reliable forecasts and the number of considered forecasts, it is easy to find that such frequencies should respectively be inferior to $0.14 \%, 1.11 \%$ and $2.16 \%$ with a level of probability of $99 \%$. The proposed forecast model is then not reliable, even if the last two frequencies are in line with the results found in [14] and [30].

Despite this weakness, this model is considered as acceptable. The first reason is based on recent research made on DLR forecasts, which indicate that the performances of the model are close to the models currently used by the TSOs. Secondly, the reliability criterion is here used to evaluate the model, but there is no evidence in the literature that this parameter is the most important one regarding DLR forecasts, and other criteria as the sharpness might be more important. As an example, with the traditional use of a fixed quantile, it might be considered that a TSO might prefer to use $1 \%$ quantile forecasts from a non-reliable but sharp forecast model than $2 \%$ quantile forecasts from another model, more reliable but less sharp. Such questions are still not answered, and they are to be addressed by using simultaneously DLR forecasts with different statistical properties and economic models providing an evaluation of the value of the forecasts. In future work, the development of the tool presented in this paper based on the use of lookup tables aims at realizing such a research of the required properties of DLR forecast models.

\section{CASe Study}

\section{A. Network Description}

The IEEE 24-bus grid is considered here (Fig. 5), with the same characteristics as the ones in [31]. It is split into two areas, each of which is considered with different load values.

The reserve allocation and activation costs are set similarly to [18]: the costs for up and down reserve allocation, $\pi$ hup $\mathrm{g}_{\mathrm{g}}$ and $\pi h d o_{g}$, are set as equal to $6 € / M W h$ for every generator $\mathrm{g}$; the reserve activation costs are set as equal to the fuel price for the down-reserve activation, and as equal to $150 \%$ of the fuel price for the up-reserve activation.

The computations of DC optimal power flows are carried out using PyPower [32], and the security constraints are added by generating constraints obtained with Benders cuts. It is considered that no line failures could occur on line 8-7, this part of the grid being operated in a radial configuration.

Lines 8-9 and 8-10 are considered to be equipped with DLR functionality, and to have exactly the same DLR value at each time. These two lines are indeed connected from bus 8 to buses 9 and 10, the latter two being at the same position. The reason for choosing these two lines is the fact that they appear to be congested when computing the Total Transfer Capacity from area 1 to area 2.

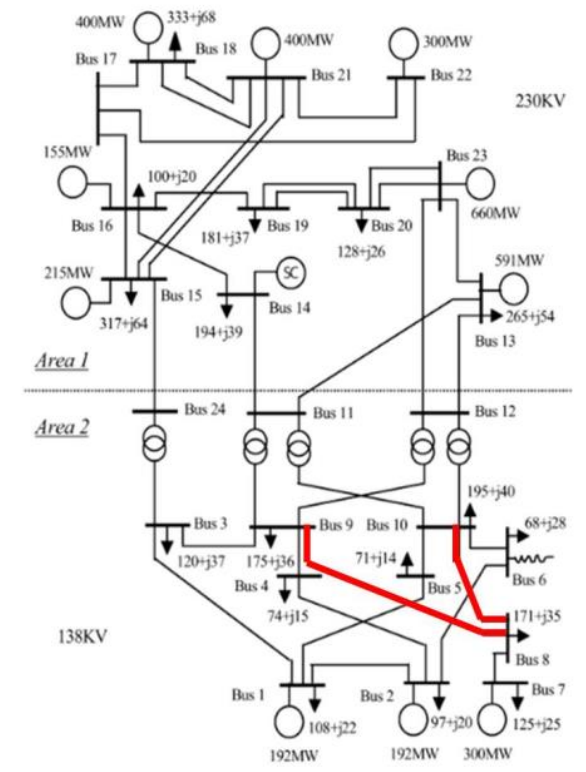

Fig. 5. IEEE 24-bus reliability test system. The lines equipped with DLR are marked in red.

Lines 8-9 and 8-10 are considered to be equipped with DLR functionality, and to have exactly the same DLR value at each time. These two lines are indeed connected from bus 8 to buses 9 and 10, the latter two being at the same position. The reason for choosing these two lines is the fact that they appear to be congested when computing the Total Transfer Capacity from area 1 to area 2 .

The line dynamic ampacity is set as equal to its initial value provided in the grid data, multiplied by the DLR divided by the SLR value. For this study, it cannot be below its nominal value. Three SLR values are used, one for each season, and are set such as to below the observed DLR for $99 \%$ of the time for the year 2009. The seasons are defined as winter (December to February), spring/autumn (March to May and September to November) and summer (June to August). Different values of SLR are defined for each weather station.

\section{B. Load Series}

In most studies involving grid simulations using DLR forecasts, only a given configuration of the grid is used. However, the grid configuration has a significant impact on the DLR impact and the selection of the optimal forecast.

We therefore propose generating load time series with a bootstrap process, considering only congestion situations, and 
taking the frequencies of the different situations from a study of historical time series. ENTSO-E data for the year 2010 are exploited for this goal. Historical data from France (area 1) and Germany (area 2) are used, since these data feature a high variety of congestion issues for the bootstrap process.

\section{RESULTS}

In order to evaluate the value of the different strategies, the cost reductions from the initial total system costs, $\mathrm{L}_{\mathrm{SLR}}(€)$, to the final total system costs obtained when DLR forecasts are used, $L_{D L R}(€)$, are considered as benefits. They are normalized by dividing them by the total $\mathrm{L}_{S L R}$ costs associated with the situations where SLR is used:

$$
\text { Benefits }=100 \% \cdot \frac{\mathrm{L}_{\mathrm{SLR}-} \mathrm{L}_{\mathrm{DLR}}}{\mathrm{L}_{\mathrm{SLR}}}
$$

In addition to the benefits, financial losses and risks are also evaluated, using three indices:

1) The frequency of events requiring reserve activations due to overestimations of DLR forecasts (\%).

2) The total cost of reserve activation $C_{\text {res act }}(€)$ for the studied period. As with the benefits, this cost is normalized using the total costs of the system when the SLR is used:

$$
\mathrm{C}_{\text {res act, } \mathrm{n}}=100 \% \cdot \frac{\mathrm{C}_{\text {res act }}}{\mathrm{L}_{\mathrm{SLR}}}
$$

3) The frequency of errors with a cost above a defined threshold f_reserve(threshold) (\%). The idea behind this is to address the fact that TSOs may aim at maintaining the DLR error magnitude below a certain threshold most of the time. This is especially the case when the reserve may be hard to call, which could be considered as when it is costly. The threshold is arbitrarily defined equal as to $€ 1500$. Situations involving such events are qualified as incidents.

\section{A. Traditional Strategies}

A first study is made by considering traditional methods, i.e. the use of fixed quantiles and risk-neutral strategies. Three strategies are tested:

1) Low arbitrary quantile $\tau$ of $1 \%$ is selected.

2) High arbitrary quantile $\tau$ of $20 \%$ is selected.

3) The value of the DLR forecast is dynamically set to minimize the expected operational costs, with a riskneutral strategy.

Regarding the choice of the quantile of $20 \%$, it is to stress that when the selected quantile becomes superior to $20 \%$, the benefits converge toward a value of $0.97 \%$. This is shown on Figure 3, where the evolution of the observed benefits with the value of a fixed selected quantile is represented.

The results shown on Figure 3 are to be compared to [33], where a similar figure is provided. Contrary to the Figure 3, [33] shows that benefits do not converge but drop sharply when quantiles reach values superior to $20 \%$. This difference in behaviour between the results presented here and [33] is due to the fact that the grid characteristics are considered in our study, and that when DLR forecast values increase above a given threshold no further improvements are observed on the grid, either because the congestions have been erased or because other congestions have appeared. These other congestions could be new congestions on other lines or changes in congestion natures from thermal limitations to stability or voltage drop limitations [34].

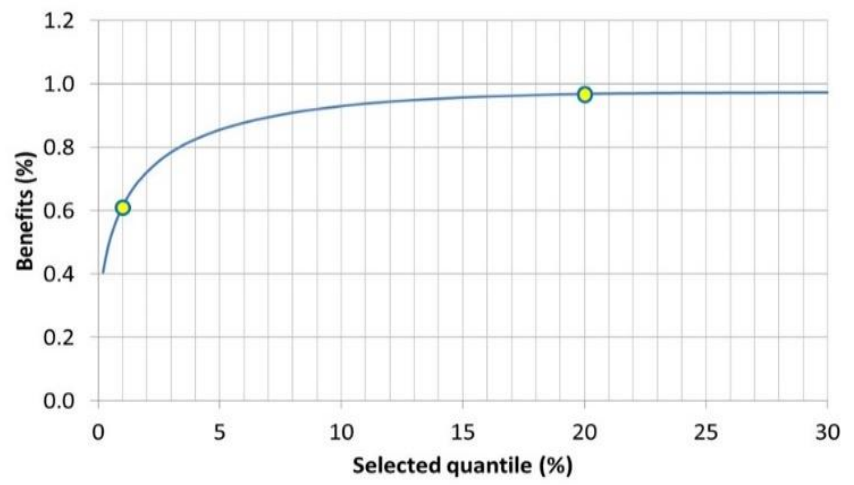

Fig. 6. Evolution of the benefits as a function of the values of the selected fixed quantiles. The two yellow circles are associated to the use of fixed quantiles equal to $1 \%$ and $20 \%$.

The observed benefits are computed for the studied period and shown on Table I, with the associated risk and loss indices associated being also shown.

The expected benefits, which are equal to the average of the expected benefits for the whole set of resolved optimization problems, were also computed, and for every situation considered in this paper, we found that the relative difference between the expected and observed benefits was less than $2 \%$. This is not surprising, considering the high amount of observations used and the fact that the stochastic part of the benefits, which is the total costs of the activated reserves, is lower than $10 \%$ and $3 \%$ of the total benefit, respectively with the use of risk-neutral optimization and a $1 \%$ quantile DLR forecast. We can thus consider that the expected and observed benefits are equal. This allows us to compare forecast models and DLR forecast setting strategies by setting the same levels of observed benefits, which are considered as known because equal to the expected benefits.

\section{TABLE I}

STRATEGY COMPARISON: OBSERVED BENEFITS, FREQUENCY OF ERRORS AND FREQUENCY OF INCIDENTS.

\begin{tabular}{|l|c|c|c|c|}
\hline $\begin{array}{l}\text { Strategy } \\
\text { used }\end{array}$ & $\begin{array}{c}\text { Benefit } \\
(\%)\end{array}$ & $\begin{array}{c}\text { Overestimation } \\
\text { frequency (\%) }\end{array}$ & $\begin{array}{c}\text { Reserve } \\
\text { costs (\%) }\end{array}$ & $\begin{array}{c}\text { f_reserve(1500€) } \\
(\%)\end{array}$ \\
\hline SLR & 0.00 & 0.00 & 0.000 & 0.00 \\
\hline$\tau=1 \%$ & 0.61 & 0.72 & 0.016 & 0.17 \\
\hline$\tau=20 \%$ & 0.97 & 4.17 & 0.105 & 1.30 \\
\hline $\begin{array}{l}\text { Risk-neutral } \\
\text { strategy }\end{array}$ & 1.01 & 3.62 & 0.085 & 1.10 \\
\hline $\begin{array}{l}\text { Perfect } \\
\text { forecast }\end{array}$ & 1.10 & 0.00 & 0.000 & 0.00 \\
\hline
\end{tabular}

Several results are noteworthy. We start by comparing the results with existing literature. Regarding levels of benefits, we consider study [5], where DLR is considered for dispatching on an IEEE 30-bus grid and benefits of around 1\% of the operational costs are found. Here, as shown in Table I, we find 
benefit values ranging from $0.2 \%$ to $1.1 \%$. Regarding total operational costs, we find a mean value of $40 \mathrm{k} €$ per hour.

Results comparable to the literature are also found if we consider the ratio between the activated reserve costs and the benefits. For a risk-neutral approach, the ratio between reserve costs $(0.085 \%)$ and benefits $(1.01 \%)$, i.e. a ratio of 1 to 12 , is close to that found in [22].

Compared to the use of $1 \%$ quantile forecasts, the use of a high-quantile (i.e. $\tau=20 \%$ ) or risk-neutral strategy increases the benefits by $60 \%$, going from $0.61 \%$ to $0.97 \%$ and $1.01 \%$ respectively. Unfortunately, this is linked with a seven-fold increase of the charges paid by the TSO and of the number of large accidents (expressed by the last column in Table I).

In terms of benefits, a dynamic risk-neutral strategy provides slightly better benefits compared to using high quantiles but a quite significant reduction (by $\sim 15 \%$ ) of the number of incidents (from $1.3 \%$ down to $1.1 \%$ ).

Thus, the dynamic selection of a level of probability for the forecast brings advantages compared to a fixed quantile. However, although the required activated amount of reserve is reduced, it is still far higher than that resulting from the use of a conservative DLR forecast (i.e. $\tau=1 \%$ ), with the TSO's charges and the number of incidents respectively multiplied by 5 (for the risk-neutral strategy) and 7 (for $\tau=20 \%$ ).

\section{B. Evaluation with Risk-Averse Strategies}

In this second part, DLR forecast are calculated as before (optimised fixed quantile $\tau$ and variable quantile) with the difference that the expected revenue function is filtered with the $\mathrm{v}_{\beta}$ function described in equation (8-10).

The evolution of operational cost linked to the DLR is followed by modifying the values of the probability $\tau$ and the parameter $\beta$. The values of $\beta$ are selected with the following numerical sequence:

$$
\beta_{\mathrm{k}}=10^{\frac{k-\alpha}{20}}
$$

For each function $v_{\beta}$, the parameters $\alpha$ and the amount of $\beta$ values are set such that for the first 5 and last 5 problems solved, the benefits observed do not vary.

In Fig. 7, for each level of benefits observed with the variations of $\beta$, the value of the total DLR forecast error costs is represented.

As we can see, for each level of benefits, linear penalties generate significant improvements in terms of reducing the total error costs. On the other hand, no improvement is observed with the use of quadratic penalties whilst the application of exponential penalties results in a worsening of the performance.
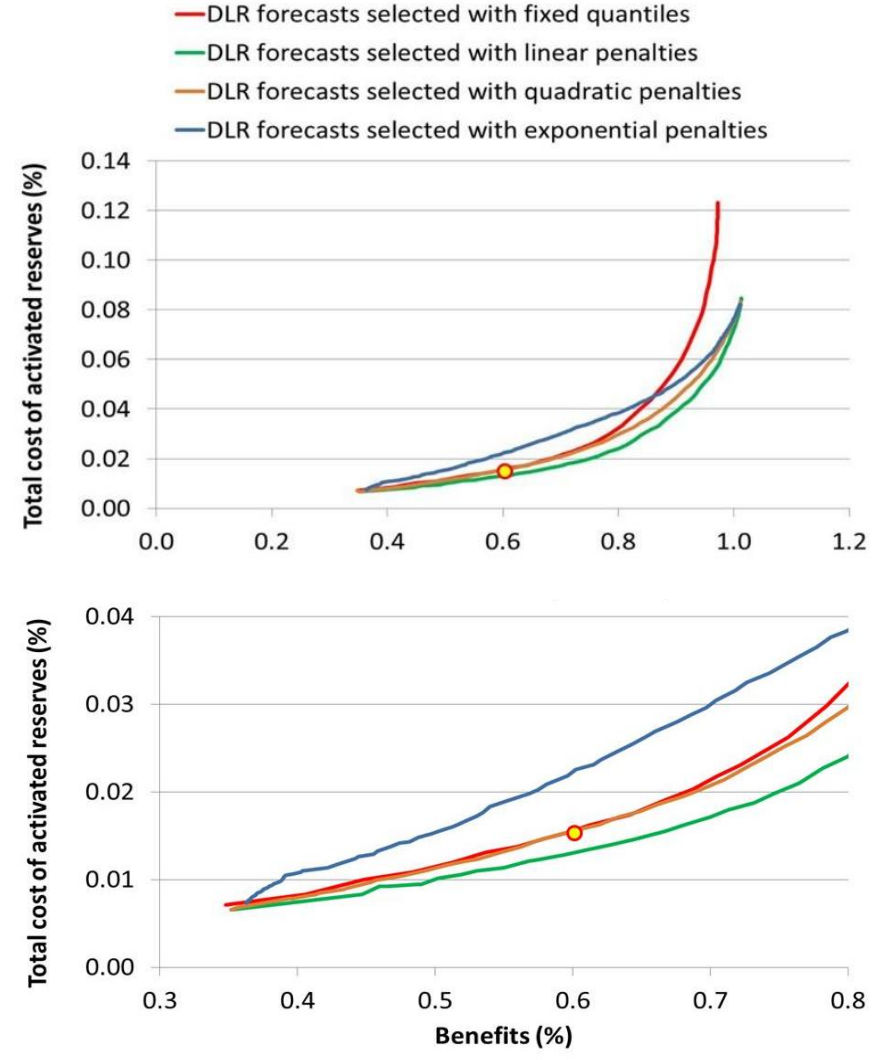

Fig. 7. Total error DLR forecast costs in function of economic benefits, depending on the use of a fixed quantile (red) or a dynamic selection, set with linear penalties (green), quadratic penalties (orange) and exponential penalties (blue). The yellow circle is associated with the use of $1 \%$ DLR quantile forecasts. The lower figure is a zoom of the upper one for the benefits in the area between $0.3 \%$ and $0.8 \%$.

Fig. 8 shows the evolution of the frequency of incidents with the observed benefits.

In contrast to what we observed with the evolution of total costs, ranking the different risk-averse strategies is complex here. Although better results are observed with the use of quadratic and exponential penalties compared to linear penalties, this ranking is not as clear as that observed in Fig. 7, especially for benefit levels close to those associated with the use of $1 \%$ quantile forecasts. This is mainly due to the quality of DLR forecasts for very low quantiles $(\leq 1 \%)$. Whereas linear penalties penalize the different potential overestimates with linear weights, and thus are only slightly sensitive to the quality of extreme quantile forecasts, this is not the case with quadratic and exponential penalties. The forecasts used in this paper have been designed to provide results similar to those presented in the literature, with no focus on the quality of the forecasts for probability levels lower than $1 \%$. It is thus not surprising that the quadratic and exponential strategies perform poorly for benefit levels associated with the use of low-quantile DLR forecasts $(\approx 0.61 \%)$. 

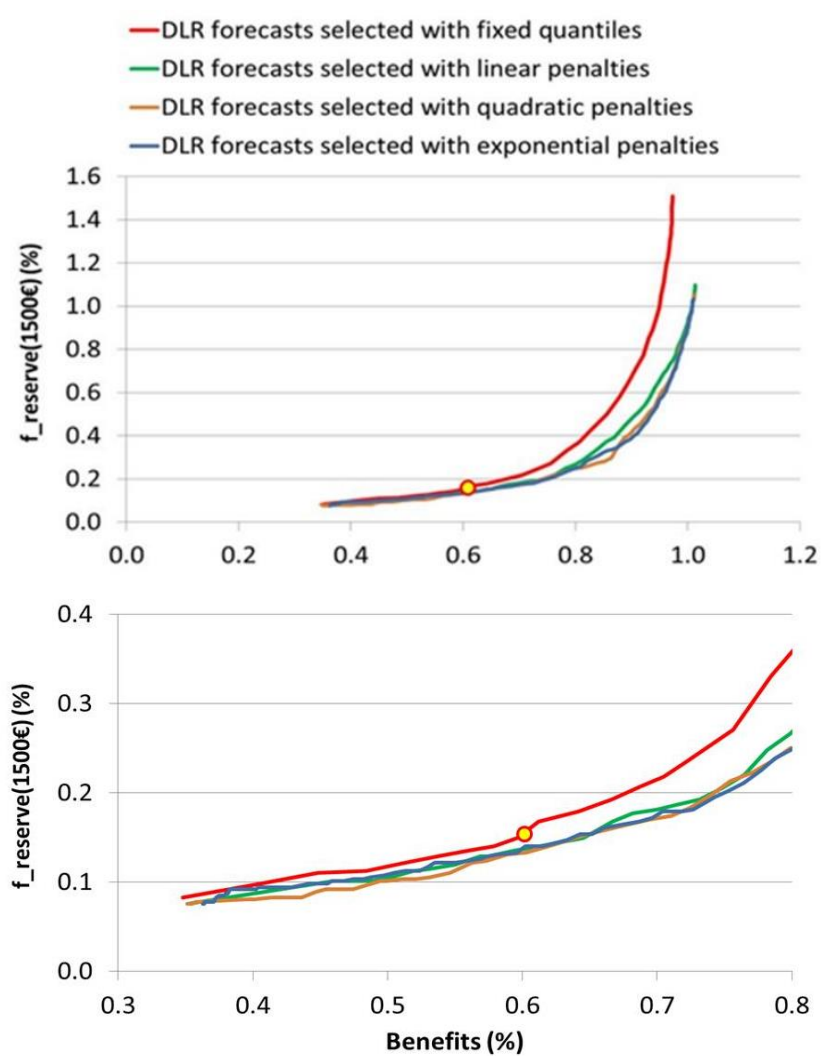

Fig. 8. Frequency of incidents in function of economic benefits, depending on the use of a fixed quantile (red) or a dynamic selection, set with linear penalties (green), quadratic penalties (orange) and exponential penalties (blue). The yellow circle is associated with the use of $1 \%$ DLR quantile forecasts. The lower figure is a zoom of the upper one for the benefits in the area between $0.3 \%$ and $0.8 \%$.

\section{CONCLUSIONS}

Recently, the use of two-stage stochastic optimization problems for setting DLR forecasts has been investigated in the literature. However, most of these studies propose risk-neutral strategies, in which the DLR forecast setting only aims at optimizing social welfare, and the TSO's risk aversion is not considered. As a result of this last point, a TSO may still prefer to continue setting DLR forecasts using fixed quantiles, thus disregarding the potential benefits of more developed strategies.

In this paper, we have proposed new methods for setting DLR using DLR probabilistic forecasts and the solving of a bilevel stochastic optimization problem, taking into account a risk aversion function. The results are promising: while ensuring low costs for the TSO or a low frequency of incidents, the benefits associated with DLR forecasts could be relatively improved to the order of $20 \%$.

This work opens up several research topics. First, the proposed methodology uses DLR quantile forecasts with probability levels below $1 \%$. Such DLR forecasts have not been investigated in the literature to date, and the interest of such forecasts is demonstrated here. Secondly, the methodology requires several forecasts of load and energy production, and a frame would need to be defined for such applications with other stochastic features. Finally, we have shown that the choice of penalty function has an important impact on the final result. For the illustration of the potential benefits, evaluation indices and penalty functions have been arbitrary defined. Further work should be carried out in the future to define them so as to reflect in a more realistic way the the objectives of a specific TSO.

\section{ACKNOWLEDGEMENT}

The European Centre for Medium-Range Weather Forecasts (ECMWF) is acknowledged for the provision of the Numerical Weather Predictions. The authors would like to thank Prof. M. Matos of INESC TEC for our useful discussions.

\section{REFERENCES}

[1] W. CIGRE, 12, Thermal Behaviour of Overhead Conductors, Tech. Brochure, 2002.

[2] "IEEE Standard for Calculating the Current-Temperature Relationship of Bare Overhead Conductors," IEEE Std 738-2012 Revis. IEEE Std 738-2006 - Inc. IEEE Std 738-2012 Cor 1-2013, pp. 1-72, Dec. 2013.

[3] T. O. Seppa, "Guide for selection of weather parameters for bare overhead conductor ratings," CIGRE WG B, vol. 2, 2006.

[4] M. W. Davis, "A new thermal rating approach: The real time thermal rating system for strategic overhead conductor transmission lines - Part I: General description and justification of the real time thermal rating system," IEEE Trans. Power Appar. Syst., vol. 96, no. 3, pp. 803-809, May 1977.

[5] M. A. Bucher, M. Vrakopoulou, and G. Andersson, "Probabilistic N-1 security assessment incorporating dynamic line ratings," in IEEE Power Energy Society General Meeting, Vancouver, Canada, 2013.

[6] M. Khaki, P. Musilek, J. Heckenbergerova, and D. Koval, "Electric power system cost/loss optimization using dynamic thermal rating and linear programming," in Electric Power and Energy Conference (EPEC), 2010 IEEE, Halifax, Canada, 2010.

[7] C. J. Wallnerström, Y. Huang, and L. Söder, "Impact From Dynamic Line Rating on Wind Power Integration," IEEE Trans. Smart Grid, vol. 6, no. 1, pp. 343-350, Jan. 2015.

[8] D. M. Greenwood and P. C. Taylor, "Investigating the Impact of RealTime Thermal Ratings on Power Network Reliability," IEEE Trans. Power Syst., vol. 29, no. 5, pp. 2460-2468, Sep. 2014.

[9] A. Michiorri et al., "Forecasting for dynamic line rating," Renew. Sustain. Energy Rev., vol. 52, pp. 1713-1730, Dec. 2015.

[10] I. Albizu, E. Fernandez, A. J. Mazon, K. J. Sagastabeitia, M. T. Bedialauneta, and J. G. Olazarri, "Overhead line rating forecasting for the integration of wind power in electricity markets," in 2015 International Conference on Clean Electrical Power (ICCEP), Taormina, Italy, 2015, pp. 382-388.

[11] H.-M. Nguyen, J.-L. Lilien, and P. Schell, "Dynamic line rating and ampacity forecasting as the keys to optimise power line assets with the integration of res. The European project Twenties Demonstration inside Central Western Europe," presented at the CIRED, Stockholm, Sweden, 2013, p. 0946.

[12] H.-M. Nguyen, J.-J. Lambin, F. Vassort, and J.-L. Lilien, “Operational experience with Dynamic Line Rating forecast-based solutions to increase usable network transfer capacity," Proc. 45th Sess. Counc. Large Electr. Syst. CIGRE, 2014.

[13] H. E. Hoekstra, C. P. J. Jansen, J. Hagen, J. W. Van Schuylenburg, J. S P. Wisse, and W. J. Zitterrsteijn, "Weather forecasted thermal line rating model for the Netherlands," in CIGRE Session, Paris, France, 2012 .

[14] T. Ringelband, P. Schäfer, and A. Moser, "Probabilistic ampacity forecasting for overhead lines using weather forecast ensembles.," Electr. Eng., vol. 95, no. 2, 2013.

[15] J. L. Aznarte and N. Siebert, "Dynamic Line Rating Using Numerical Weather Predictions and Machine Learning: A Case Study," IEEE Trans. Power Deliv., vol. 32, no. 1, pp. 335-343, Feb. 2017.

[16] "DLR Forecasting Presented at FERC - Nexans." [Online]. Available: http://www.nexans.us/eservice/US-en_US/navigatepub_0_32903/DLR_Forecasting_Presented_at_FERC.html. [Accessed: 08May-2018].

[17] M. A. Bucher and G. Andersson, "Robust Corrective Control Measures in Power Systems With Dynamic Line Rating," IEEE Trans. Power Syst., vol. 31, no. 3, pp. 2034-2043, May 2016.

[18] Y. Chen, F. Teng, R. Moreno, and G. Strbac, "Impact of dynamic line rating with forecast error on the scheduling of reserve service," in 
Power and Energy Society General Meeting (PESGM), Boston, USA, 2016.

[19] K. Kopsidas, A. Kapetanaki, and V. Levi, "Optimal Demand Response Scheduling With Real-Time Thermal Ratings of Overhead Lines for Improved Network Reliability," IEEE Trans. Smart Grid, vol. 8, no. 6, pp. 2813-2825, 2017.

[20] B. Banerjee, D. Jayaweera, and S. M. Islam, "Alleviating postcontingency congestion risk of wind integrated systems with dynamic line ratings," in Power Engineering Conference (AUPEC), 2014 Australasian Universities, Perth, Australia, 2014.

[21] B. Banerjee, S. M. Islam, and D. Jayaweera, "Monte Carlo based method for managing risk of scheduling decisions with dynamic line ratings," in Power \& Energy Society General Meeting, Denver, USA, 2015.

[22] F. Teng, R. Dupin, A. Michiorri, G. Kariniotakis, Y. Chen, and G. Strbac, "Understanding the Benefits of Dynamic Line Rating under Multiple Sources of Uncertainty," IEEE Trans. Power Syst., vol. 33, no. 3, pp. 3306-3314, 2018

[23] B. Banerjee, D. Jayaweera, and S. M. Islam, "Optimal scheduling with dynamic line ratings and intermittent wind power," in PES General Meeting| Conference \& Exposition, 2014 IEEE, National Harbor, USA, 2014.

[24] M. Miura, T. Satoh, S. Iwamoto, and I. Kurihara, "Application of dynamic rating to evaluation of ATC with thermal constraints considering weather conditions," in 2006 IEEE Power Engineering Society General Meeting, Montreal, Canada, 2006.

[25] N. Meinshausen, "Quantile Regression Forests," J. Mach. Learn. Res., vol. 7, no. Jun, pp. 983-999, 2006.

[26] Y. Zhang and J. Wang, "GEFCom2014 probabilistic solar power forecasting based on k-nearest neighbor and kernel density estimator," in IEEE Power Energy Society General Meeting, Denver, USA, 2015.

[27] T. Chen and C. Guestrin, "Xgboost: A scalable tree boosting system," in Proceedings of the 22nd acm sigkdd international conference on knowledge discovery and data mining, 2016, pp. 785-794.

[28] G. Ke et al., "Lightgbm: A highly efficient gradient boosting decision tree," in Advances in Neural Information Processing Systems, 2017, pp. 3146-3154.

[29] J. Snoek, H. Larochelle, and R. P. Adams, "Practical bayesian optimization of machine learning algorithms," in Advances in neural information processing systems, 2012, pp. 2951-2959.

[30] X. Sun, P. B. Luh, K. W. Cheung, and W. Guan, "Probabilistic forecasting of dynamic line rating for over-head transmission lines," in IEEE Power Energy Society General Meeting, Denver, USA, 2015.

[31] C. Grigg et al., "The IEEE Reliability Test System-1996. A report prepared by the Reliability Test System Task Force of the Application of Probability Methods Subcommittee," IEEE Trans. Power Syst., vol. 14, no. 3, pp. 1010-1020, Aug. 1999.

[32] R. Lincoln, PyPower. 2017.

[33] R. Dupin, A. Michiorri, and G. Kariniotakis, "Dynamic line rating dayahead forecasts - cost benefit based selection of the optimal quantile," presented at the CIRED Workshop, Helsinki, Finland, 2016, pp. 122126.

[34] L. Dawson and A. Knight, "Applicability of Dynamic Thermal Line Rating for Long Lines," IEEE Trans. Power Deliv., vol. 33, no. 2, pp. 719-727, 2018.

Romain Dupin is a graduate of Ecole Centrale Lille, where he took a Chair of Electrical Networks option, and from the University of Lille 1, where he gained a Master's degree in electrical engineering in 2014. Since September 2014, he has been a PhD student at the MINES ParisTech Center for Processes, Renewable Energies and Energy Systems (PERSEE) in Sophia Antipolis, France. He works on developing methods for forecasting dynamic line rating and studies the impacts of dynamic line rating on power system management.

Andrea Michiorri received an Eng. degree in Mechanical Engineering with a specialization in Energy from the University of Rome 'La Sapienza' in 2005. $\mathrm{He}$ then obtained a PhD from the University of Durham in 2010 with a dissertation on the thermal state estimation of power system components. He is currently an associate professor at the MINES-ParisTech Center for Processes, Renewable Energies and Energy Systems (PERSEE) in Sophia Antipolis, France, working on the integration of renewable resources and distributed generators into the power system, with a particular focus on the aspects of decision-making under uncertainty.

George Kariniotakis (S'95-M'02-SM'11) was born in Athens, Greece. He received his Eng. and M.Sc. degrees from Greece in 1990 and 1992 respectively, and his PhD from Ecole des Mines de Paris in 1996. He currently works at the MINES ParisTech PERSEE Centre as a senior scientist and head of the Renewable Energies and Smart Grids Group. He has authored more than 220 scientific publications in journals and conferences. He has been involved as participant or coordinator in more than $40 \mathrm{R} \& \mathrm{D}$ projects in the fields of renewable energies and distributed generation. Among them, he was the coordinator of some major EU projects in the field of wind power forecasting such as Anemos, Anemos.plus and SafeWind projects. His scientific interests include among others timeseries forecasting, decision making under uncertainty, modeling, management and planning of power systems. 